\title{
Identifying local and descending inputs for primary sensory neurons
}

\author{
Yi Zhang, ${ }^{1}$ Shengli Zhao, ${ }^{1}$ Erica Rodriguez, ${ }^{1}$ Jun Takatoh, ${ }^{1}$ Bao-Xia Han, ${ }^{1}$ Xiang Zhou, ${ }^{2}$ and Fan Wang ${ }^{1,3}$ \\ 'Department of Neurobiology, Duke University Medical Center, Durham, North Carolina, USA. ²Department of Biostatistics, University of Michigan, Ann Arbor, Michigan, USA. \\ ${ }^{3}$ Department of Cell Biology, Duke University Medical Center, Durham, North Carolina, USA.
}

\begin{abstract}
Primary pain and touch sensory neurons not only detect internal and external sensory stimuli, but also receive inputs from other neurons. However, the neuronal derived inputs for primary neurons have not been systematically identified. Using a monosynaptic rabies viruses-based transneuronal tracing method combined with sensory-specific Cre-drivers, we found that sensory neurons receive intraganglion, intraspinal, and supraspinal inputs, the latter of which are mainly derived from the rostroventral medulla (RVM). The viral-traced central neurons were largely inhibitory but also consisted of some glutamatergic neurons in the spinal cord and serotonergic neurons in the RVM. The majority of RVM-derived descending inputs were dual CABAergic and enkephalinergic (opioidergic). These inputs projected through the dorsolateral funiculus and primarily innervated layers I, II, and V of the dorsal horn, where pain-sensory afferents terminate. Silencing or activation of the dual CABA/enkephalinergic RVM neurons in adult animals substantially increased or decreased behavioral sensitivity, respectively, to heat and mechanical stimuli. These results are consistent with the fact that both GABA and enkephalin can exert presynaptic inhibition of the sensory afferents. Taken together, this work provides a systematic view of and a set of tools for examining peri- and extrasynaptic regulations of pain-afferent transmission.
\end{abstract}

\section{Introduction}

In the somatosensory system, different types of primary sensory neurons are specialized to detect separate qualities (e.g., temperature, pain, itch, touch, and proprioception) and intensities of sensory stimuli. The peripheral axons of sensory neurons innervate the skin, muscles, joints, or internal organs, while the central afferent axons project to the spinal cord dorsal horn and terminate in different layers, depending on their sensory modalities. Generally speaking, cutaneous afferents transmitting nociceptive (painful) stimuli project to layers I, II, and V, whereas afferents transmitting innocuous touch stimuli project to layers III, IV, and V (1-3). Importantly, sensory stimuli detected by the peripheral axon terminals are not the only source of inputs received by sensory neurons (4). Using electron microscopy (EM) studies (5), it was observed that many central axons of sensory neurons in the spinal cord participate in the formation of synaptic glomeruli structures, in which the sensory afferent terminal forms the core of the glomerulus, and the terminal is both presynaptic to several dendrites and postsynaptic to axo-axonic and dendro-axonic inputs. The main neurotransmitter of these axo-axonic and dendro-axonic inputs was shown to be GABA (5-7). GABA released at these axo-axonic synapses causes primary afferent depolariza-

Note regarding evaluation of this manuscript: Manuscripts authored by scientists associated with Duke University, The University of North Carolina at Chapel Hill, Duke-NUS, and the Sanford-Burnham Medical Research Institute are handled not by members of the editorial board but rather by the science editors, who consult with selected external editors and reviewers.

Conflict of interest: The authors have declared that no conflict of interest exists. Submitted: January 25, 2015; Accepted: July 23, 2015.

Reference information: / Clin Invest. 2015;125(10):3782-3794. doi:10.1172/JCI81156. tion (PAD) that depresses action potentials arriving at the afferent terminal (8-10). Thus, these inputs onto sensory afferents are thought to control pain and touch sensory transmission through presynaptic inhibition. However, despite decades of EM studies and electrophysiological recordings of PAD, the neuronal sources and identity of these presynaptic inputs onto diverse primary afferents remain poorly understood (4).

Furthermore, nociceptive sensory processing in the dorsal spinal cord is also subject to powerful control by supraspinal sites, which was first described by Patrick Wall in a seminal paper published in 1967 (11). Specifically, the periaqueductal gray (PAG) and the rostroventral medulla (RVM) in the brainstem play critical roles in descending pain modulation (12-16). The pain modulation initiated by RVM was found to be either inhibitory or facilitatory, depending on electrical-stimulation strength (17). Based on neuronal activity associated with the nociceptive withdrawal reflex, RVM neurons can be classified as ON-cells, OFF-cells, and NEUTRAL-cells, which form the neural basis for the bidirectional control of pain by RVM (17-20). In general, ON-cells facilitate nociception and OFF-cells are antinociceptive. However, the primary targets and circuit mechanisms of the RVM descending modulation in the dorsal spinal cord remain opaque. Interestingly, there has been some indirect evidence suggesting that antinociceptive OFF-cells might provide inputs directly onto nociceptive primary afferents and suppress pain through presynaptic inhibition (21).

Since neuronal derived nonsensory inputs can substantially affect the neurotransmission of primary afferents, we decide to use an unbiased approach to systematically map the neural source of inputs onto cutaneous sensory neurons. The deficient monosynaptic rabies virus $(\Delta \mathrm{G}-\mathrm{RV})$ is an ideal tool to trace presynaptic inputs of infected neurons $(22,23)$. Previous studies showed that 
A

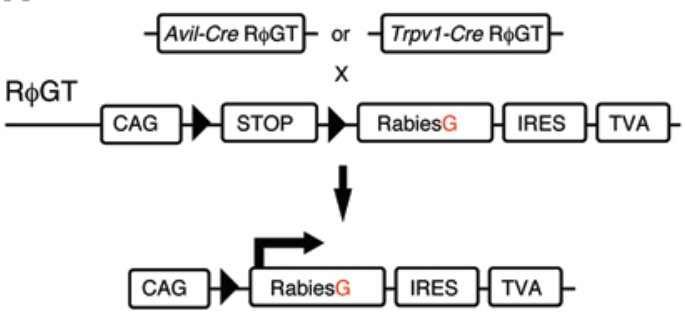

C

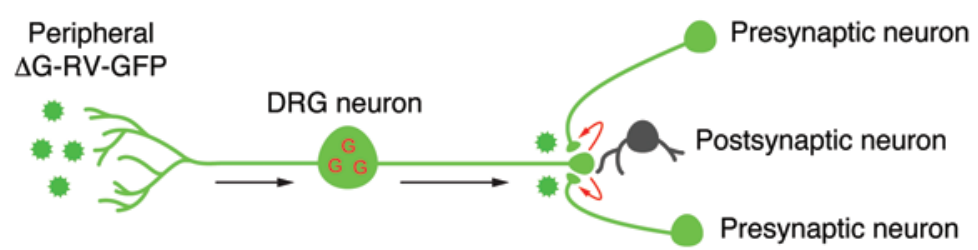

B $\triangle$ G-RV-GFP

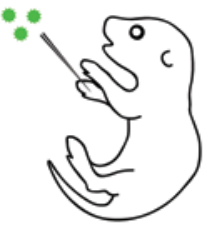

Injection Sampling

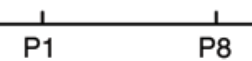

P8 Dorsal horn

Presynaptic neuron

\begin{abstract}
Figure 1. Schematic drawing of strategy for tracing presynaptic inputs onto sensory afferents. (A) Genetic crosses were used to generate Avil-Cre RФGT and Trpv1Cre RФGT mice such that the rabies $\mathrm{G}$ protein can be selectively expressed in either all or TRPV1-lineage of DRG sensory neurons. (B) $\Delta \mathrm{G}-\mathrm{RV}$-GFP was injected into the plantar skin of the right front paw of P1 pups, as illustrated. Seven days after injection, the samples are collected and analyzed. (C) Expected outcome of the viral tracing experiments: $\Delta \mathrm{G}-\mathrm{RV}$-GFP will infect DRG neurons from peripheral axon terminal and will be transported back to the cell bodies, where the deficient virus will be complemented by the rabies $G$ protein. Subsequently, some of the replicated $\Delta \mathrm{G}$-RV-GFP will be released at the central afferent terminals in the dorsal horn and infect their putative presynaptic partners.
\end{abstract}

the commonly used "fixed" strains of rabies virus could infect mouse primary sensory neurons from axon terminals, could be transported anterogradely in these neurons, and could be released from their axon terminals (24-26). We have previously developed a mouse line that enables Cre-dependent complementation of the deficient rabies virus (RФGT mouse) $(27,28)$. Here, crossing RФGT mice with 2 different Cre drivers enabled us to complement $\Delta G-R V$ either selectively in all primary sensory neurons or specifically in TRPV1-expressing nociceptive neurons, which are mostly peptidergic c-fibers (Figure 1). Using this strategy, we mapped the neural sources of inputs onto cutaneous sensory neurons. Notably, our results discovered a group of RVM-derived dual GABAergic/ enkephalinergic descending neurons that can provide inputs onto primary afferents. These neurons project to spinal layers I, II, and $\mathrm{V}$ and exert important antinociception functions.

\section{Results}

Genetic strategy for tracing inputs onto either all types or TRPV1-expressing primary sensory neurons. The RФGT mouse allows for Cre-dependent expression of the rabies- $G$ protein, as well as the TVA receptor (a protein expressed by avian species) (refs. 27,28, and Figure 1A). Crossing this conditional R $\Phi$ GT mouse with 2 different Cre drivers enables the rabies-G protein and TVA receptor to be selectively expressed in different classes of sensory neurons (Figure 1A). For most of our experiments, we used deficient rabies virus ( $\triangle \mathrm{G}-\mathrm{RV}-\mathrm{GFP})$ that can infect all types of sensory neurons independent of TVA receptor. In one set of experiments, we used EnvA-pseudotyped-deficient rabies virus (EnvA-RV), which only infects TVA-expressing neurons (EnvA binds TVA). The EnvA-RV has a lower titer than that of $\Delta \mathrm{G}-\mathrm{RV}$ and thus lower infectivity (see Methods); therefore, most of experiments were performed using $\Delta \mathrm{G}-\mathrm{RV}$. Two Cre driver lines, advillin-Cre (AvilCre) and Trpv1-Cre, were crossed with RФGT mice in this study. AvilCre is expressed by all primary sensory neurons $(29,30)$, whereas Trpv1-Cre is mainly expressed in peptidergic nociceptive neurons and in some nonpeptidergic c-fiber neurons (due to transient expression of TRPV1 in nonpeptidergic neurons) (31).

We injected $\Delta \mathrm{G}-\mathrm{RV}-\mathrm{GFP}$ into the plantar skin of the right front paw of P1 pups of 3 different genotypes: (i) RФGT without Cre (as a control); (ii) Avil-Cre RФGT; and (iii) Trpv1-Cre RФGT. $\Delta \mathrm{G}-\mathrm{RV}$ should be able to enter into the peripheral axon terminals of cutaneous neurons in all genotypes but should only be complemented in Cre-expressing DRG neurons. In other words, $\Delta \mathrm{G}-\mathrm{RV}$ can be complemented in any infected DRG neurons in Avil-Cre RФGT or only in nociceptive TRPV1-lineage neurons in Trpv1-Cre RФGT mice. In theory, complemented viruses will spread into the neurons that potentially provide inputs onto the infected DRG neurons (Figure 1C). Seven days after injection at P8, we collected the spinal cords and brains, and examined the patterns of viral spreading.

Intraganglionic release and spreading of rabies virus. The cell bodies of primary sensory neurons reside in the dorsal root ganglia (DRG). We dissected the cervical and thoracic DRG (C5 to T2 segment) and the corresponding spinal cords from viral-injected mice. We first examined infection in DRG. Unexpectedly, the numbers of labeled sensory neurons varied dramatically depending on the genotypes (Figure 2). First - in the absence of the Cre transgene - on average, about $558 \pm 65$ sensory neurons ( $n=3$ mice) in total were labeled, and there were no labeled cells observed in the spinal cord and brainstem (Figure 2, A and D, and Table 1), suggesting no leaky spreading of the $\Delta \mathrm{G}-\mathrm{RV}$ without rabies-G protein complementation. By contrast, in Avil-Cre RФGT and Trpv1-Cre RФGT mice, the average numbers of infected DRG neurons were increased dramatically to 3,669 $\pm 351(n=3)$ and 2,326 $\pm 366(n=3)$, respectively (Figure $2, \mathrm{~B}$ and $\mathrm{C}$, and Table 1 ). Since the same amount (200 nanoliters) of $\Delta \mathrm{G}-\mathrm{RV}-\mathrm{GFP}$ was injected into all mice, the large differences in the number of labeled DRG neurons could be best explained by the fact that, after complementation, there was intraganglion spreading of the rabies virus from infected neurons to neighboring DRG neurons. In other words, in RФGT mice without Cre expression, no cells can complement the virus, and the approximately 550 labeled DRG neurons were results of direct infection. In Trpv1-Cre RФGT mice, Cre is expressed in subsets of neurons; therefore, only some of the $\Delta \mathrm{G}-\mathrm{RV}$-infected neurons could complement the deficient virus. However, in Avil-Cre RФGT mice, Cre is expressed by all DRG neurons - thus, all infected neurons can complement the virus. Consequently, through intraganglion spreading of the replicated viruses, more DRG neurons were labeled in Avil-Cre RФGT mice $(23,600)$ than in Trpv1-Cre RФGT mice $(\sim 2,300)$. 


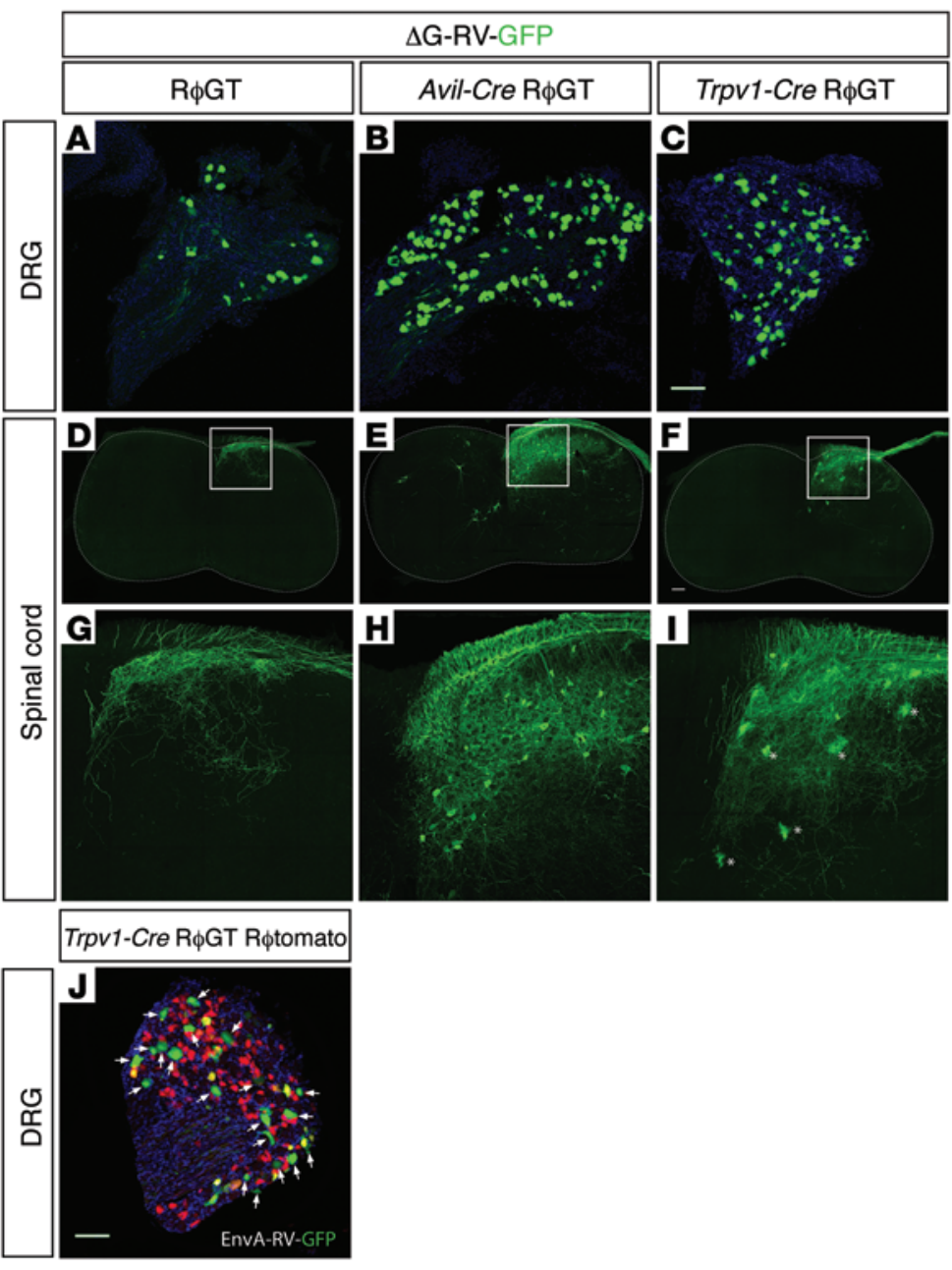

Figure 2. $\Delta$ G-RV-GFP-labeled cells in DRG and the spinal cord. (A-C) Representative images of $\triangle \mathrm{G}$-RV-GFP-labeled neurons in DRG (cervical segment C6 or C7) from RФGT (control), Avil-Cre RФGT, or Trpv1-Cre RФGT mouse. Scale bar, $100 \mu \mathrm{m}$. (D-F) Representative images of $\Delta \mathrm{G}$-RV-GFP-labeled neurons in the cervical spinal cord from RФGT (control), Avil-Cre RФGT, or Trpv1-Cre RФGT mouse. Scale bar, $100 \mu \mathrm{m}$. (G-I) Magnified view of the boxed areas in D-F. Asterisks indicate glial cells. (J) Representative image of EnvA-RV-GFP-labeled neurons in DRG from Trpv1-Cre RФGT RФtomato mouse. Arrows indicate GFP-single positive neurons.

To further confirm the intraganglion spreading of rabies virus, we injected the EnvA-pseudotyped virus (EnvA-RV-GFP) into Trpv1-Cre RФGT RФtomato pups (Figure 2J). In this case, Trpv1-Cre induced the expression of rabies-G, TVA receptor, and also tomato fluorescent protein in these neurons, and only these neurons can be infected by EnvA-RV-GFP (through a TVA receptor). In DRG isolated from these mice after EnvA-RV-GFP injection into the forepaw, we observed tomato ${ }^{+}$-only (no GFP, uninfected), $\mathrm{GFP}^{+} /$tomato $^{+}$(double positive), as well as $\mathrm{GFP}^{+}$ only (no tomato) neurons (Figure 2J). The $\mathrm{GFP}^{+}$-only neurons are not Trpv1-Cre neurons; therefore, they must have received the replicated virus secondarily from the primary infected TRPV1expressing (tomato ${ }^{+}$) neurons. This experiment confirmed intraganglion spreading of rabies after complementation. These results, together with other previous studies, highlight the extensive presence of intraganglion neuron-neuron communications (see Discussion).
Spreading of monosynaptic rabies virus from sensory neurons into the spinal cord revealed local interneurons that potentially provide inputs onto primary afferents. We next examined serial sections through spinal cords from the 3 groups of mice. As mentioned above, there were no labeled neurons in spinal cords from viral-injected RФGT mice in the absence of Cre (Figure 2, D and G). In AvilCre RФGT mice, since over 3,600 primary DRG neurons were infected with $\triangle \mathrm{G}$-RV-GFP (either through direct infection or through secondary intraganglion infection), the sensory axons projecting into the dorsal horn were extensively labeled by GFP (Figure 2, E and $\mathrm{H}$ ). In addition to afferent axons, many spinal neurons were labeled across multiple segments (Figure 2, E and H), with a total of $1,712 \pm 260$ spinal neurons per animal $(n=3$, Table 1 , Supplemental Video 1; supplemental material available online with this article; doi:10.1172/JCI81156DS1). Supplemental Video 1 shows the entire serial sections of all labeled neurons throughout one spinal cord. Most of the labeled spinal neurons were found in layers II-V ipsilateral to the injection side, with a few scattered neurons on the contralateral side (Figure 2E).

In contrast, in Trpv1-Cre RФGT mice - although 2,326 \pm 366 primary DRG neurons were infected by the virus we only observed, on average, $85 \pm 22$ labeled neurons in the entire spinal cord (average of $n=3$ mice, Table 1 and Supplemental Video 2). Supplemental Video 2 shows the entire serial sections through the spinal cord to give an overview of the sparse labeling in these mice. There were also some labeled glial cells (with morphology resembling either protoplasmic astrocyte or microglial cells) scattered in the spinal cord (marked by stars in Figure 2I). However, the number of labeled glial cells varied randomly across sections and among animals. To determine roughly how many of the approximately 2,300 DRG neurons were Trpv1-Cre-expressing cells, we injected $\Delta \mathrm{G}-\mathrm{RV}-\mathrm{GFP}$ virus into Trpv1-Cre RФGT RФtomato mice and found that $59.5 \% \pm 2.1 \%$ of all infected DRG neurons were tomato ${ }^{+}$ (Supplemental Figure 1). Thus, about 1,300 labeled neurons were TRPV1 ${ }^{+}$and could potentially complement the virus, as opposed to approximately 3,600 in Avil-Cre RФGT mice (2.7-fold difference). Yet the number of labeled spinal neurons in Trpv1-Cre RФGT mice were 20-fold less than that observed in AvilCre RФGT (85 versus 1,700; Table 1). Since Trpv1-Cre is expressed primarily in peptidergic nociceptive neurons, the limited labeling of spinal neurons corroborates the fact that peptidergic afferents rarely participate in synaptic glomeruli structures $(5,32)$ and

\section{Table 1. Quantification of labeled DRG and spinal neurons in mouse of the 3 different genotypes}

Genotypes

Number of DRG neurons

Number of SC neurons

\section{RФGT}

$(n=3)$

$558 \pm 65$
Avil-Cre RФGT $(n=3)$
$3,669 \pm 351$
$1,712 \pm 260$

Trpv1-Cre RФGT

$(n=3)$

$2,326 \pm 366$

$85 \pm 22$ 
In situs for ipsilateral-labeled spinal cord neurons in Avil-Cre RфGT
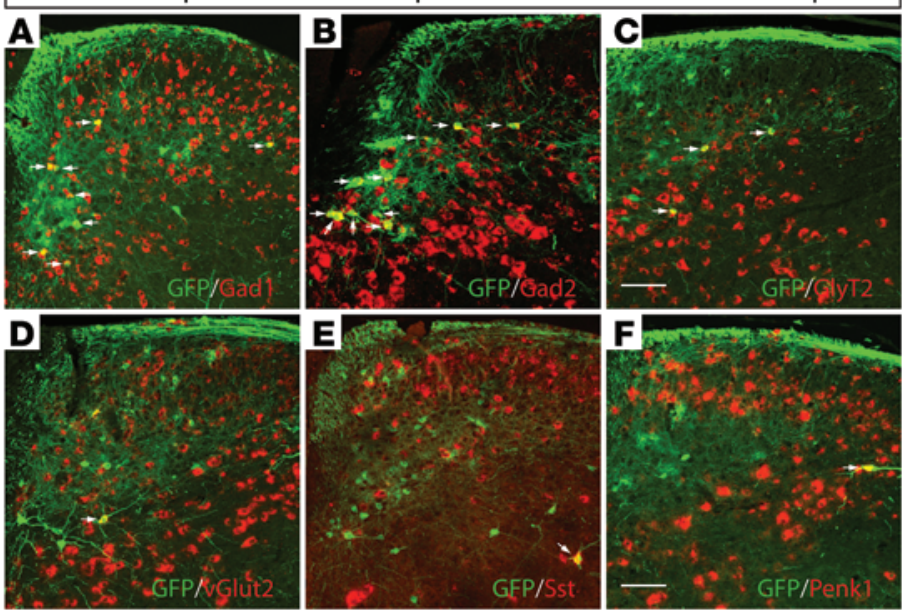

In situs for contralateral-labeled spinal cord neurons in Avil-Cre R $\phi \mathrm{GT}$
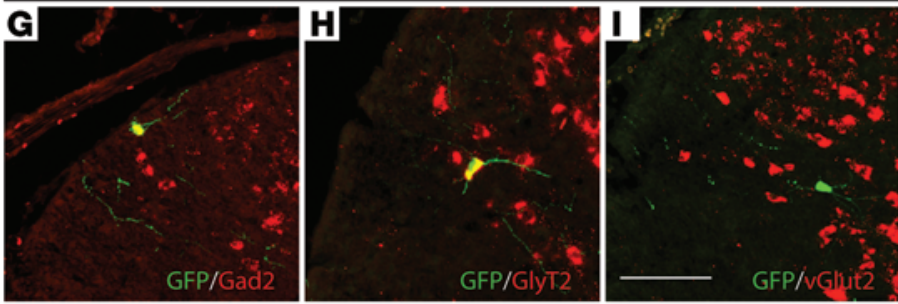

Figure 3. Molecular characterizations of $\triangle$ G-RV-GFP-labeled spinal cord neurons. Representative images from the 2-color in situ hybridization (red signals) and immunostaining (green, anti-GFP) analyses of $\triangle \mathrm{G}$-RV-GFP-labeled spinal neurons in Avil-Cre RФGT mice are shown. Arrows indicate some of the $\mathrm{GFP}^{+}$neurons expressing the molecular marker examined. (A-F) Images from ipsilateral spinal cord are shown. The in situ probes used are GAD1 (A), GAD2 (B), GlyT2 (C), vGlut2 (D), SST (E) and PENK1 (F). Scale bar, $100 \mu \mathrm{m}$. (G-I) Images from contralateral spinal cord with in situ probes of GAD2 (G), GlyT2 (H), and vGlut2 (I) are shown. Scale bar, $100 \mu \mathrm{m}$. receive many fewer direct presynaptic inputs. In other words, the lack of terminals making synapses directly with infected TRPV1 ${ }^{+}$ afferents likely led to the failure of rabies spreading into the spinal cord. This result is consistent with the known property of the rabies virus as a retrograde presynaptic transsynaptic tracer that enters into neurons from presynaptic terminals (33).

We further performed molecular characterization of labeled spinal neurons in Avil-Cre RФGT mice using in situ hybridization with a variety of neuronal transmitter and modulator markers as probes. About $45.5 \%$ of labeled spinal interneurons are $\mathrm{GAD}^{+}$, $44.4 \%$ are $\mathrm{GAD}_{2}{ }^{+}$(both GAD1 and GAD2 are markers for GABAergic neurons), and $47.1 \%$ are GlyT2 ${ }^{+}$(vesicular glycine transporter 2, a marker for glycinergic neurons), suggesting that the majority of inputs received by sensory afferents are inhibitory in nature (Figure 3, A-C and G-I, and Table 2). Somewhat surprisingly, approximately $20 \%$ of the total labeled neurons are vGlut2 ${ }^{+}$(Figure 3D and Table 2; see Discussion for implications of this result and alternative explanations). We also examined several other markers - somatostatin (SST), neuronal nitric oxide synthase (NOS1), preproenkephalin (PENK1), and thyrotropin-releasing hormone (TRH) - to determine whether the labeled spinal interneurons belong to a specific subtype. SST, NOS1, and PENK1 were known to be expressed in subsets of neurons in the dorsal spinal cord and in some GABAergic neurons in the brain (34-37). TRH was known to express in subsets of neurons in the spinal cord and in RVM, and was shown to have a short, strong antinociceptive activity against chemical and mechanical stimuli (38); NOS synthesizes nitric oxide (NO), which can modulate inflammatory pain (39). While TRH and NOS1 are largely absent in labeled spinal neurons, $14.4 \%$ and $18.2 \%$ of the viral-labeled neurons are $\mathrm{SST}^{+}$ (Figure 3E) or PENK1+ (Figure 3F), respectively (Table 2).
Rabies-mediated tracing also revealed descending inputs from RVM dual GABAergic/enkephalinergic neurons to primary sensory afferents. Interestingly, when we examined the brains from the $\Delta G$-RV-GFP tracing experiments, we observed a few labeled neurons in the ipsilateral cuneate nucleus, brainstem reticular regions (medullary, lateral, and parvocellular reticular region), vestibular nucleus, spinal trigeminal nucleus, and the red nucleus (Supplemental Figure 2). Interestingly, we consistently labeled about 100 neurons per animal in the RVM in all Avil-Cre RФGT samples (96 \pm 33 per animal, $n=3)$ and a few ( 5 per animal, $n=3)$ in Trpv1Cre RФGT samples (data not shown) (Figure 4). Since rabies virus

\section{Table 2. Molecular marker analysis of labeled neurons in the spinal cord and RVM}

$\begin{array}{lcc}\text { Markers } & \begin{array}{c}\text { \% of labeled neurons } \\ \text { in spinal cord }\end{array} & \begin{array}{c}\text { \% of labeled neurons } \\ \text { in RVM }\end{array} \\ \text { CAD1 } & 45.5(299 / 657) & \text { ND } \\ \text { CAD2 } & 44.4(715 / 1,611) & 61.5(48 / 78) \\ \text { ClyT2 } & 47.1(721 / 1,530) & 28.6(20 / 70) \\ \text { vGlut2 } & 21.0(349 / 1,664) & 0.0(0 / 65) \\ \text { TPH2 } & 0.0(0 / 529) & 17.3(9 / 52) \\ \text { CAD2 + ClyT2 + TPH2 } & \text { ND } & 100(83 / 83) \\ \text { SST } & 14.4(82 / 571) & 1.5(1 / 66) \\ \text { NOS1 } & 3.3(20 / 609) & 0.0(0 / 57) \\ \text { PENK1 } & 18.2(100 / 550) & 66.8(96 / 146) \\ \text { TRH } & 0.0(0 / 511) & 0.0(0 / 58)\end{array}$

The percentages of infected neurons expressing molecular markers per total GFP' neurons are shown. The numbers in parentheses are in situ positive cells/GFP+ neurons. ND, not determined. 


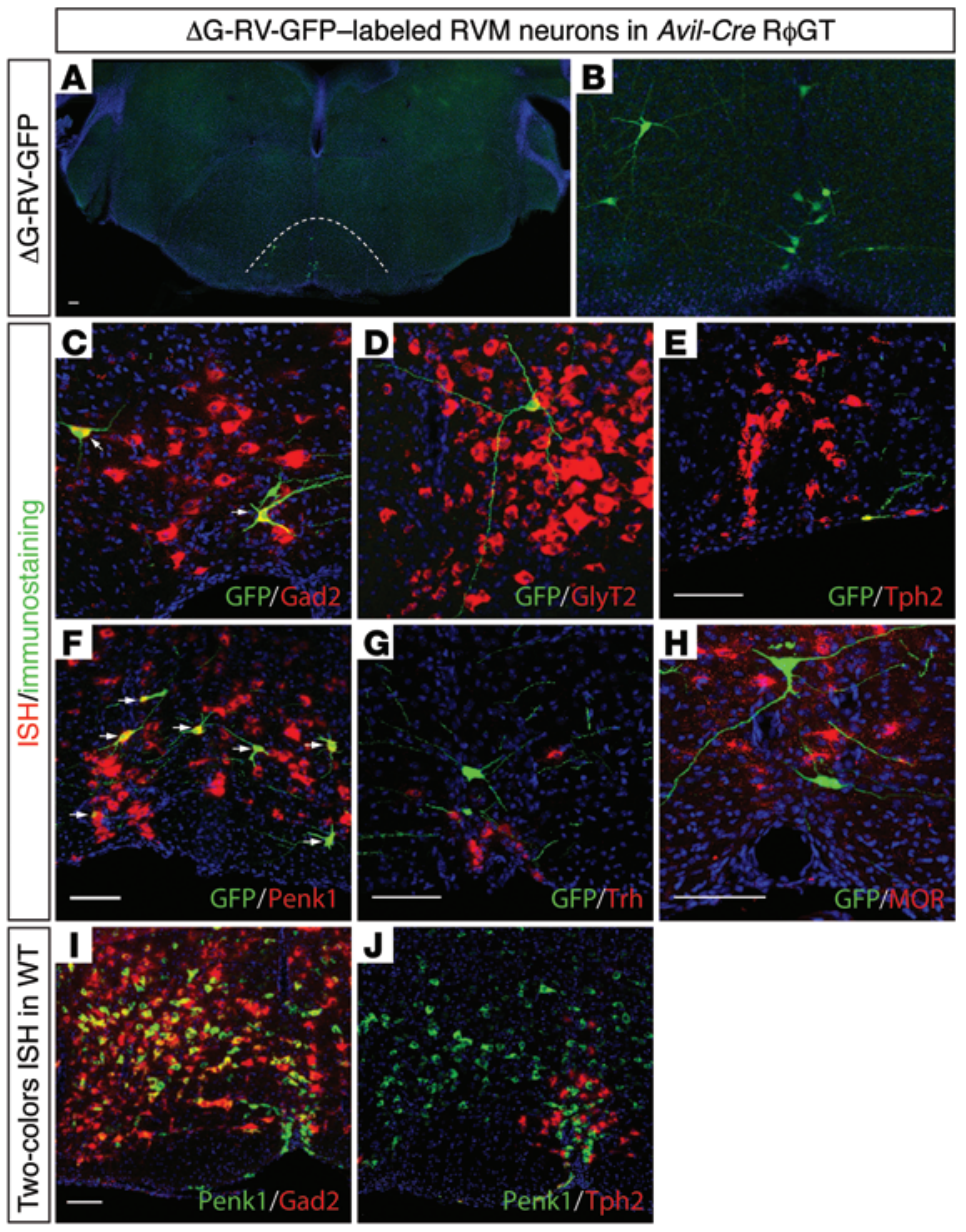

Figure 4. Analyses of $\triangle \mathbf{G}-\mathbf{R V}$-GFP-labeled neurons in RVM. (A and B) Representative images of $\triangle \mathrm{G}$-RV-GFP-labeled RVM neurons in Avil-Cre RФGT mice. Enlarged image of labeled neurons in $\mathbf{A}$ is shown in $\mathbf{B}$. (C-H) Representative images from in situ hybridization (ISH) analyses of $\triangle \mathrm{G}$-RV-GFP-labeled RVM neurons. The probes used are GAD2 (C), GlyT2 (D), TPH2 (E), PENK1 (F), TRH (C), and MOR (H). (I and J) Representative 2-color in situ experiments that examine the coexpression of PENK1 and GAD2 (I), and PENK1 and TPH2 (J) in RVM region. Scale bar for all images is $100 \mu \mathrm{m}$. Arrows indicate double-labeled neurons.

only spread into $10 \% \sim 30 \%$ of all presynaptic neurons (22), and the labeling of long-distance inputs is even less efficient, the labeled RVM neurons should be a minor fraction of the actual population of such neurons. We therefore focused on the labeled RVM neurons.

Molecular characterization of rabies-labeled RVM neurons in Avil-Cre RФGT pups revealed that $61.5 \%$ express GAD2, $28.6 \%$ express GlyT2, 17.3\% express TPH2 (a marker for serotonergic neurons), and none express vGlut2 (Figure 4 and Table 2), indicating RVM inputs onto primary afferents are either serotonergic (5-HT; 17\%) or GABA/Glycinergic ( 80\%) (Figure 4 and Table 2). In fact, when we used a mixture of GAD2/GlyT2/TPH2 probes, all viral-labeled neurons were positive for the mixed probes. We refer to the RVM neurons that putatively provide inputs onto primary afferents as RVM-pre cells.

Viral-labeled RVM-pre cells rarely coexpress SST, NOS1, or TRH (<3\%) (Table 2). By contrast, about $66.8 \%$ of labeled RVMpre neurons coexpress PENK1 (Figure $4 \mathrm{~F}$ and Table 2). Since there were also roughly the same amount of RVM-pre neurons express- ing GAD2 (61.5\%), this raised the possibility that the majority of the nonserotonergic RVM-pre neurons coexpress GAD2 and PENK1. To confirm this, we performed 2-color in situ hybridization in P8 animals. Indeed, more than $80 \%$ of $\mathrm{PENK} 1^{+}$neurons coexpress GAD2 in RVM (Figure $4 \mathrm{I}$; there are also many GAD2 single-positive neurons). In contrast, only $2 \%$ of PENK $1^{+}$neurons coexpress TPH2, or vGlut 2 in RVM (Figure 4J and data not shown). Since Penk1 gene products encode enkephalins (the endogenous opioid ligand), RVM-pre cells therefore could provide dual GABAergic and enkephalinergic inputs onto primary afferents and other target neurons in the spinal cord.

Furthermore, a previous study had discovered that RVM pronociceptive ON-cells express the $\mu$-opioid receptor (MOR) (40). We therefore examined the expression of MOR in viral-labeled RVM-pre neurons using in situ hybridization. We found that none of the rabies-labeled neurons expressed MOR at this stage (Figure $4 \mathrm{H}$ ), suggesting the dual GABAergic/enkephalinergic RVM-pre neurons may not be ON-cells. However, others have found that in acute RVM slices, MOR is expressed in many types of neurons (41), so it is not a definitive marker of identified projection neurons.

The GABAergic/enkephalinergic RVM-pre neurons innervate dorsal spinal cord in adult mice. The discovery that many RVM-pre neurons express PENK1 in our neonatal tracing experiments provided molecular tools to examine the projection pattern of these neurons in the adult. We first cloned a conserved 726-bp genomic sequence just upstream of the first exon of the Penk1 gene and used it as a promoter to drive Cre expression (see Methods). We generated a lentivirus carrying the Penk1-Cre transgene and coinjected the LentiPenk1-Cre with AAV-CAG-FLEX-GFP into the RVM regions of about 6- to 8-week-old WT mice. If the dual GABAergic/ enkephalinergic neurons persist into adult, this strategy should label these neurons in the adult RVM. Three weeks after the viral injection, we sectioned the brain (Figure 5A) and examined whether $\mathrm{GFP}^{+}$neurons also express Penk1 or Gad 2 mRNA, and this experiment should also test the specificity of the short Penk1 promoter. Double immunofluorescence/in situ labeling experiments showed that more than $50 \%$ of $\mathrm{GFP}^{+}$cells (driven by Lenti-Penk1-Cre) also express Penk1 mRNA, and similarly, more than $50 \% \mathrm{GFP}^{+}$cells are also GAD2 ${ }^{+}$(Figure 5 , B and C). Less than $2 \%$ of $\mathrm{GFP}^{+}$neurons are vGlut $2^{+}$or $\mathrm{TPH} 2^{+}$, and the rest $(\sim 30 \%)$ of the $\mathrm{GFP}^{+}$cells are glial cells near the injection site (data not shown). Thus, the Lenti-Penk1-Cre labeled PENK1 $1^{+} / \mathrm{GAD} 2^{+}$neurons in adult RVM (although it also leads to ectopic expression in glial cells). Importantly, GFP-labeled RVM axons projected through the dorsal-lateral funiculus (DLF) along the entire length of the spinal cord (Supplemental Figure 3 and Figure 5, E and H). Collaterals from these RVM axons showed extensive innervation in 2 regions of the dorsal horn: a superficial region and the lamina $\mathrm{V}$ (Figure 5, E and H, and Supplemental Figure 3, C, F, and I). Using coimmunostaining of anti-vGlut1, which labels terminals from touch afferents in Lamina III-IV, we found that GFP-labeled RVM axons sparsely innervated lamina III-IV (Figure 5, D-F, Supplemental Figure 3, $\mathrm{H}-\mathrm{J}$ ). Using IB4 staining, which labels nonpeptidergic afferent ter- 

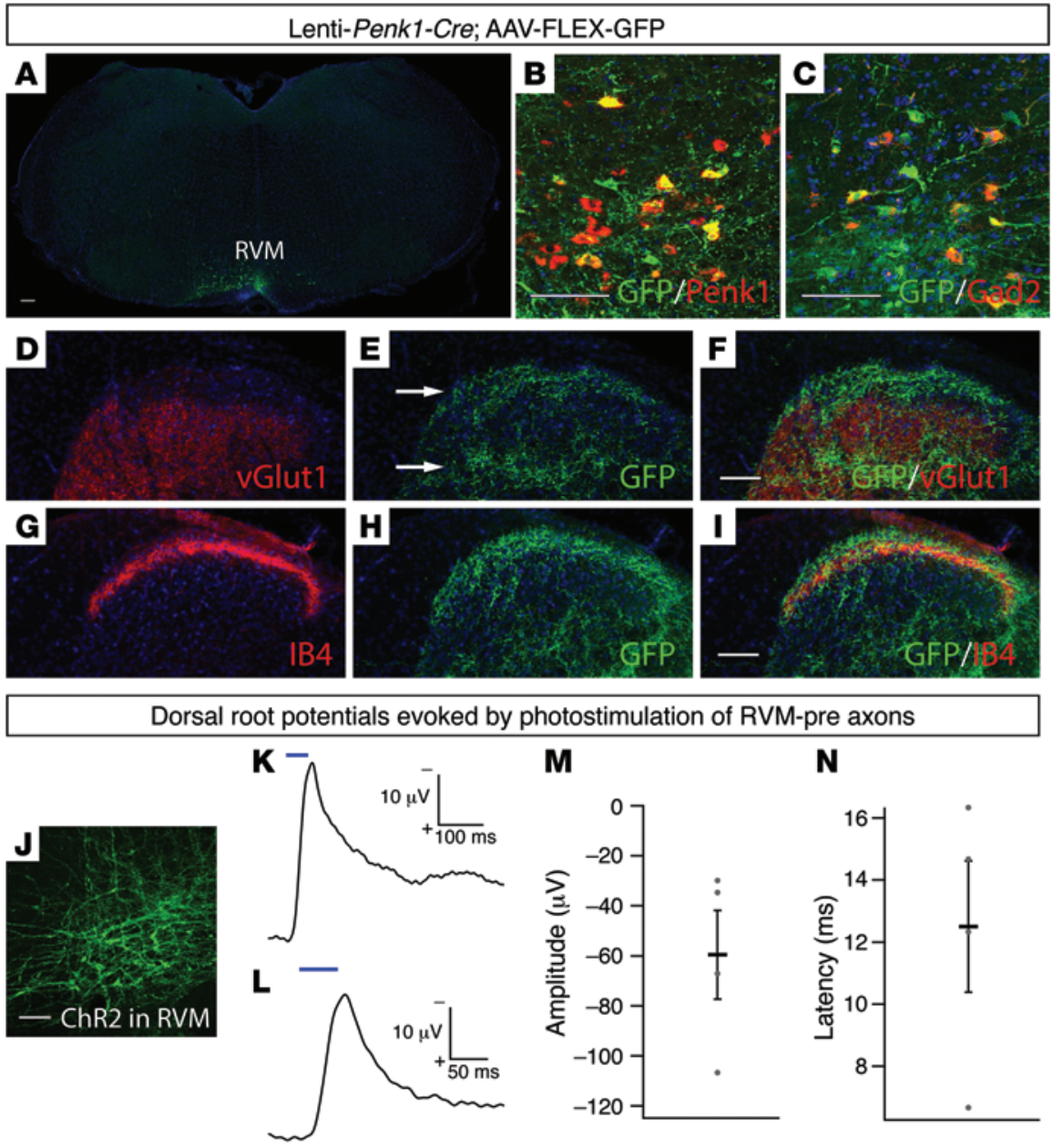

\section{M}

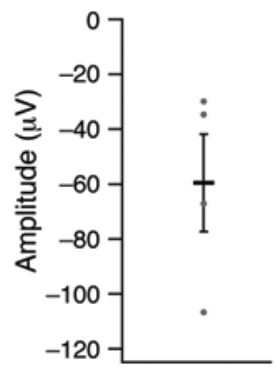

minals in lamina II, we found that labeled RVM axons innervate both lamina II and lamina I located above the IB4 ${ }^{+}$layer (Figure 5, G-I, and Supplemental Figure 3, B-G). This lamina-termination pattern (I, II, and V) is consistent with previously reported patterns of RVM neuron projections (42-44).

To obtain further evidence that dual GABAergic/enkephalinergic RVM neurons can provide inputs to sensory afferents in adult mice, we expressed channelrhodopsin (ChR2) (45) in these neurons by coinjecting Lenti-Penk1-Cre with AAV-EF1a-FLEX-ChR2eYFP into the adult RVM (Figure 5J). As mentioned above, due to the high intracellular chloride concentrations in the sensory afferent, GABA causes PAD, which gives rise to dorsal root potentials (DRPs) $(46,47)$. We therefore examined whether photoactivation of the ChR2 $2^{+}$RVM axons, which should result in the release of GABA, could produce DRP (see Methods). Indeed, photostimulation evoked DRPs with short latencies (average amplitude $59.6 \pm$ $17.7 \mu \mathrm{V}$, average latency $12.5 \pm 2.1 \mathrm{~ms}$, recorded from a cervical dorsal root, $n=4$ ) (Figure $5, \mathrm{~K}-\mathrm{N}$ ), suggesting that the labeled RVM-pre neurons provide functional presynaptic inputs to primary afferents.

Effects of silencing the GABAergic/enkephalinergic RVM-pre neurons in adult mice. It is known that nociceptive c-fibers project to lamina I and II, and nociceptive A $\delta$ fibers project to lamina I, II, and $\mathrm{V}(1,2)$. Thus, inputs from the dual GABAergic/enkephalinergic RVM-pre neurons (either through axo-axonic synapses or through extrasynaptic spillover) onto nociceptive afferents in these layers

\section{$\mathbf{N}$}

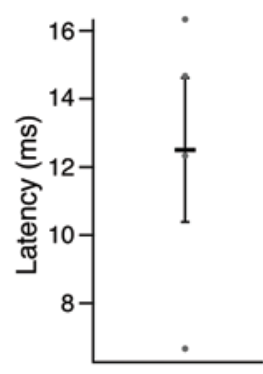

Figure 5. RVM-pre neurons project axons to dorsal spinal cord, and their activation evokes DRPs. (A) Representative images of labeled RVM neurons from mice coinjected with LentiPenk1-Cre and AAV-FLEX-GFP. (B and C) In situ hybridization showed that RVM neurons labeled by coinjection of Lenti-Penk1-Cre and AAV-FLEXGFP express PENK1 (B) and/or GAD2 (C). (D-F) Costaining with anti-vGlut1 (marker for touch afferents) revealed that labeled RVM axons are concentrated in 2 regions (arrows): a superficial region above the vGlut1+ layers and the deep vGlut1+ lamina V. (G-I) Costaining with IB4 (marker of lamina II) revealed that labeled RVM axons innervate both lamina I and II. (J) Representative image of ChR2 expression in RVM neurons after coinjection of Lenti-Penk1-Cre and AAV-FLEX-hChR2-eYFP in RVM. (K and $\mathbf{L}$ ) Optogenetic stimulation of ChR2-expressing axons in spinal block-evoked DRPs. Two representative traces are shown. ( $\mathbf{M}$ and $\mathbf{N}$ ) Light-evoked EPSP peak amplitude and latency were shown $(n=4$ mice). Scale bar is $100 \mu \mathrm{m}$ for all images. should exert presynaptic inhibition and, thus, antinociceptive functions. This would predict that silencing the RVM-pre neurons in adults should result in hypersensitivity to nociceptive stimuli. To test this hypothesis, we first expressed tetanus toxin light chain (TeLC) in RVM-pre neurons. Note that TeLC cleaves synaptobrevin (VAMP) proteins on synaptic vesicles $(48,49)$. Thus, while it will prevent the release of GABA, it may not affect the release of the neuropeptide enkephalin from dense core vesicles, since most of these normally do not contain VAMP $(50,51)$. Hence, this manipulation blocks GABA but not enkephalin transmission of these RVM-pre neurons.

We coinjected Lenti-Penk1-Cre and AAV-FLEX-TeLC-GFP into RVM regions of WT mice. As an independent alternative method, we also injected AAV-FLEX-TeLC-GFP into the RVM of Gad2-Cre knockin mice. Gad2-Cre is likely expressed by both descending RVM projection neurons and local inhibitory neurons in $\operatorname{RVM}(42,52)$, and thus, it is not an ideal tool. However, if many of the local GAD2 ${ }^{+}$neurons function to inhibit the descending $\mathrm{GAD2}^{+}$RVM neurons, the net effect of silencing both populations will be the same as silencing only the descending neurons. On average, about $1,517 \pm 207$ or $1,606 \pm 289$ RVM neurons $(n=3$ for Lenti-Penk1-Cre, $n=3$ for Gad2-Cre) were infected for LentiPenk1-Cre and Gad2-Cre, respectively. WT mice and mice with coinjection of Lenti-Penk1-Cre and AAV-FLEX-GFP were used as controls. When we tested the behaviors at 2 weeks after viral injec- 

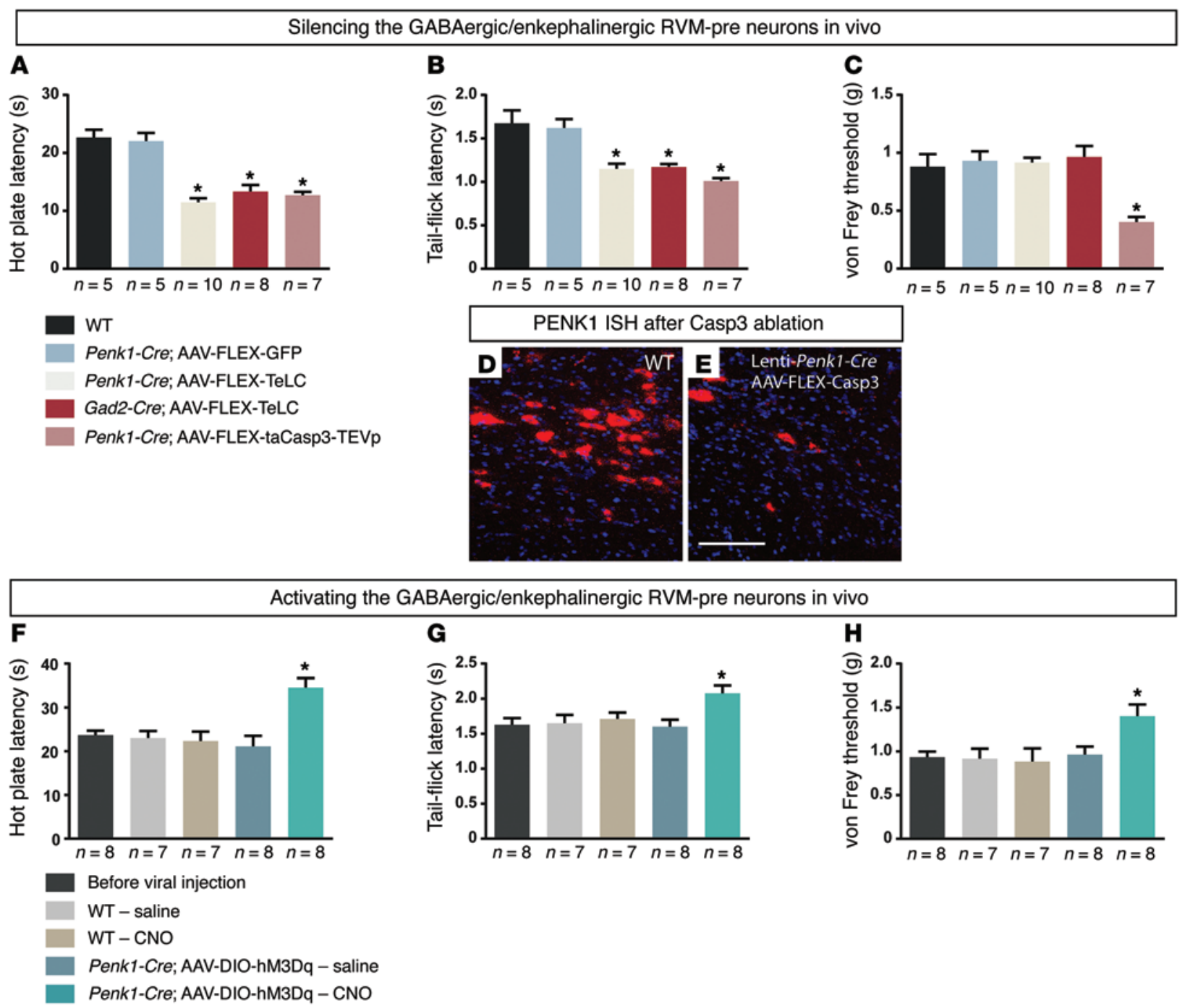

Figure 6. Behavioral effects of silencing and activating the inputs from the dual GABAergic/enkephalinergic RVM neurons. (A-C) Behavioral tests for the effects of silencing or ablating RVM-pre neurons compared with controls. Numbers ( $n$ ) of mice in each group are shown on the graphs. (A) Hot plate paw-withdraw latency, (B) tail-flick latency; and (C) paw-withdraw threshold of von Frey test. (D and E) PENK1 in situ hybridization (ISH; red) confirmed the loss of PENK1-expressing RVM neurons after coinjection of Lenti-Penk1-Cre and AAV-FLEX-taCasp3-TEVp. Control sample (D) and caspapse-ablated sample (E). (F-H) Behavioral tests for the effects of activating RVM-pre neurons compared with controls. Numbers ( $\mathrm{n}$ ) of mice in each group are shown on the graphs. Hot plate paw-withdraw latency $(\mathbf{F})$; tail-flick latency $(\mathbf{C})$; and paw-withdraw threshold of von Frey test $(\mathbf{H})$. Error bars represent means \pm SEM $P$ values represent comparison to WT and control injection values $\left({ }^{*} P<0.05\right)$. Differences were determined by Student's $t$ test between 2 groups, or oneway ANOVA followed by post-hoc Bonferroni test for multiple groups. $n=5 \sim 10$ mice.

tion, there were no significant differences between TeLC-expressing and control groups, likely because AAV-mediated expression requires 3 or more weeks to reach sufficient levels (Supplemental Figure 4). Starting 3 weeks and up to 6 weeks after viral injection, we performed thermal- and mechanical-sensitivity behavior tests (multiple trials per animal). In both hot plate and tail-flick tests, TeLC-mediated silencing of Penk1-Cre- or Gad2-Cre-expressing RVM inputs resulted in significant heat hypersensitivity compared with WT or control-injected mice (i.e., reduced latency of paw licking or tail flicking) (Figure 6, A and B). However, when we performed a von Frey test, the threshold of paw lifting was unchanged (Figure 6C). These results suggest that GABA derived from RVMpre inputs is critical for attenuating heat but not mechanical pain sensitivity (see more in Discussion).
To completely block the function of the GABAergic/ enkephalinergic RVM neurons, we performed cell-ablation experiments. Using the recently published Cre-dependent active caspase cell-ablation system (AAV5-FLEX-taCasp3-TEVp) (53), we coinjected Lenti-Penk1-Cre and AAV5-FLEX-taCasp3-TEVp into the RVM of WT mice. In situ hybridization using a Penk1 probe confirmed a substantial loss of PENK1+ neurons in RVM after caspase ablation (82.1\% reduction, $n=3$, compare Figure 6 , D and E). Behavior tests were performed 3 weeks after viral-mediated ablation. We found that ablating Penk1-Cre expressing RVM neurons resulted in both heat and mechanical hypersensitivity: i.e., reduced latency in hot plate and tail-flick responses, as well as reduced paw-withdrawal threshold in von Frey test (Figure 6, A-C, last column). Taken together with the results of the tetanus toxin, 
A

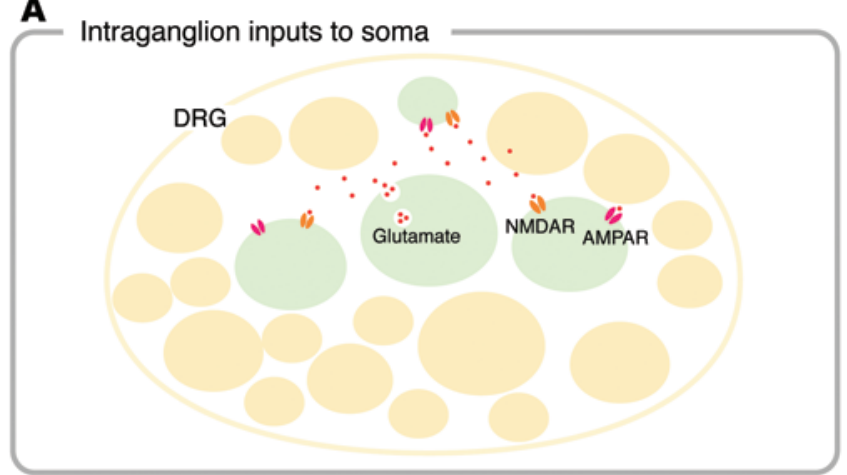

B

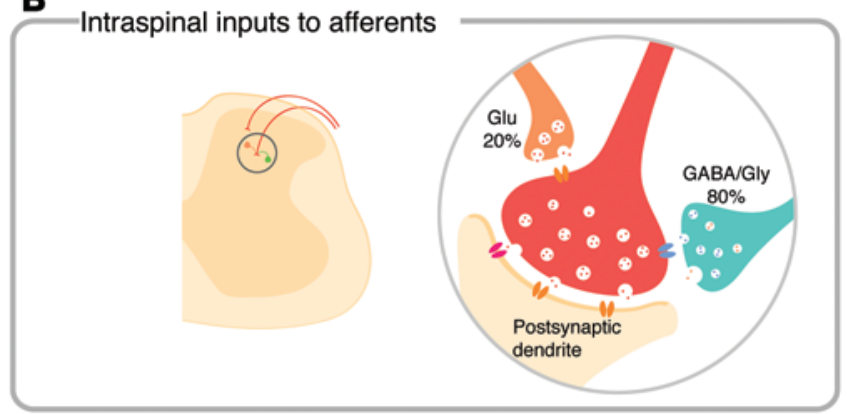

C

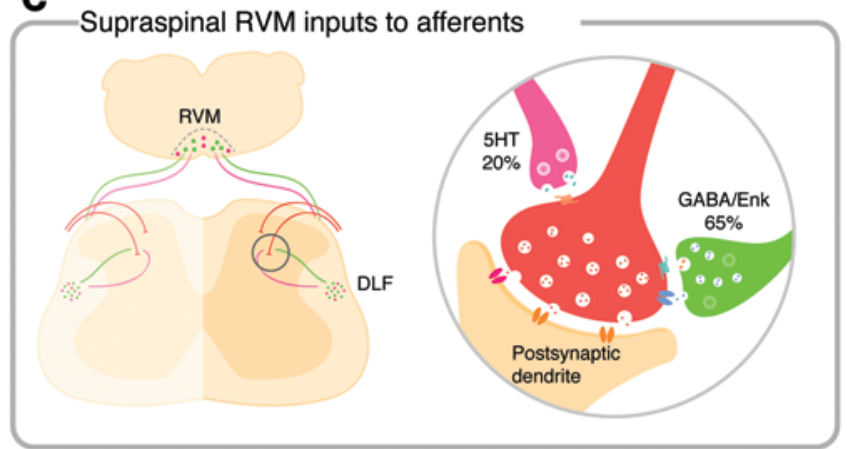

it suggests that in the absence of RVM-derived GABA, enkephalin released from RVM-pre is sufficient to maintain the mechanical nociceptive sensitivity, but removing both RVM-derived GABA and encephalin resulted in hypersensitivity to both heat and mechanical stimuli (see more in Discussion).

Effects of activating the GABAergic/enkephalinergic RVM-pre neurons in adult mice. To determine the effect of increasing the activities of the RVM-pre neurons, we expressed the chemogenetic activator hM3Dq DREADD (designer receptor exclusively activated by designer drug) in these neurons by coinjecting LentiPenk1-Cre and AAV-hSyn-DIO-hM3D(Gq) into RVM in adult WT mice (after they were tested for baseline sensitivities to thermal and mechanical stimuli). The excitatory hM3Dq receptor is exclusively activated by the ligand CNO (54). Four weeks after viral injection, we administered either saline or $\mathrm{CNO}(0.3 \mathrm{mg} / \mathrm{kg})$ in the control and in experimental groups, and we performed behavioral tests (see Methods). Saline or CNO had no effects on behaviors in WT mice, and saline did not affect mice with hM3Dq expressed in RVM-pre neurons (Figure 6, F-H). By contrast, CNO-mediated activation of the RVM-pre neurons resulted in hyposensitivity (increased threshold for response) in hot plate, tail-flick, and
Figure 7. Schematic drawing of extra- and peri-synaptic inputs onto sensory neurons revealed by this study. (A) Intraganglion inputs to sensory neuron soma. Glutamate and possibly other transmitters released from the soma of sensory neurons can potentially modulate activities of neighboring sensory neurons in an extrasynaptic manner. (B) Intraspinal inputs onto sensory afferent terminals. Certain types of spinal GABAergic (some are also glycinergic) interneurons can form axo-axonic synapses with sensory afferents, and small numbers of glutamatergic interneurons have axons (or collaterals) that locate immediately adjacent to sensory afferent terminals. These spinal neurons regulate afferent transmission either through direct synaptic inputs or through spillover. (C) Supraspinal RVM-derived inputs onto sensory afferent terminals. RVM serotonergic and dual CABA/enkephalinergic neurons provide inputs primarily onto nociceptive sensory afferents.

von Frey tests (last column in Figure 6, F-H). These results provide further support that the Penk1-Cre-labeled dual GABAergic/ enkephalinergic RVM neurons have antinociception functions.

\section{Discussion}

In this study, we used a monosynaptic rabies virus-based approach to trace inputs onto primary sensory neurons in neonatal mice. Results revealed intraganglion, intraspinal, and supraspinal (RVM) inputs that could modulate sensory transmission at periand extrasynaptic levels (see summary in Figure 7). We further examined the nature of RVM-descending innervation of primary afferents. We demonstrated that the majority of those RVMderived inputs are dual GABAergic/enkephalinergic. In adults, these RVM-pre neurons target spinal layers I, II, and V; their activities can elicit DRPs in primary afferents, and they exert important antinociception functions in acute behavioral tests.

Technical concerns: transneuronal versus trans-synaptic spreading of rabies virus from primary mouse sensory afferents. Previous tracing studies using rabies virus consistently demonstrated that peripheral uptake of rabies virus typically occurs at motor terminals and propagates retrogradely via exclusive trans-synaptic transfer into presynaptic neurons $(23,33,55)$. However, in animal species such as mice, which have a particular affinity for fixed rabies strains (due to their passage history), the virus can enter into peripheral terminals of sensory neurons and move in an anterograde manner (24-26). The question is whether the spread of rabies to other neurons from infected primary sensory neurons happens primarily with presynaptic partners or nonselectively to any nearby neurons.

On the one hand, the drastic reduction of labeled neurons in the spinal cord when rabies virus was complemented in TRPV1 ${ }^{+}$ neurons, as opposed to pan-sensory neuron complementation (in Avil-Cre mice), supports the view that rabies virus spreads primarily and most efficiently to presynaptic terminals located immediately adjacent to sensory afferents (i.e. into peri-afferent presynaptic terminals). As noted previously, TRPV1+ ${ }^{+}$peptidergic neurons rarely participate in synaptic glomeruli structures $(5,32)$. They instead receive inputs through volume transmission or spillover. Accordingly, the limited number of spinal neurons labeled from $\mathrm{TRPV}^{+}$afferents can be explained by the low probability of rabies to spread into extrasynaptic inputs.

On the other hand, our finding of intraganglion spreading of rabies virus appears to contradict this view. Interestingly, we and others have previously shown that DRG neurons release glutamate 
from their soma when activated, a process referred to as intraganglion neural transmission $(56,57)$. Basbaum and colleagues have observed intraganglion transfer of WGA between DRG neurons (58). Others observed intraganglion release of neuromodulators, such as CGRP and ATP (59-61). The release of glutamate and other transmitters from DRG neuron somas exposes presynapticlike features on the soma that can be recognized by rabies virus (55). The unusual efficient extrasynaptic intraganglion transfer of rabies virus may be the result of the densely packed cell bodies inside the ganglion, which greatly increases the chances of replicated virus to find an adjacent target (Figure 7A). Nevertheless, this result suggests that neighboring neurons in the ganglion are a source of inputs for sensory neurons. Such intraganglion inputs might be a major contributor to secondary allodynia, i.e., pain spreading to neighboring areas after injury and/or in chronic pain conditions. Finally, we noted that the viral-tracing experiments were performed in neonatal mice, and since the sensory circuits undergo maturation and changes from neonates to adults (62), some of the findings might be developmental phenomena that do not persist in the adult.

Local GABAergic and glutamatergic inputs from spinal interneurons to primary sensory afferents. In the spinal cord, we found that rabies virus crossed from sensory afferent terminals into numerous interneurons located primarily in the ipsilateral dorsal horn, with a few scattered neurons in the contralateral dorsal horn and near the central canal. This result indicates that local neurons in the dorsal spinal cord are the dominant source of inputs to primary sensory afferents. The majority (80\%) of viral-labeled neurons express markers for GAD1, GAD2, or GlyT2, consistent with previous EM studies, indicating that GABA is the main neurotransmitter at the axo-axonic synapses onto sensory afferents (Figure $7 \mathrm{~B})$. GABA induces PAD that suppresses action potentials arriving at the afferent terminals, resulting in presynaptic inhibition of sensory transmission (8-10).

Somewhat unexpectedly, a minority $(20 \%)$ of labeled spinal interneurons expressed vGlut $2^{+}$, indicating that they were glutamatergic. Since no previous studies reported glutamatergic axo-axonic synapses onto primary afferents, this result suggests 2 possibilities. First, these glutamatergic inputs are extrasynaptic, located in the vicinity of sensory afferents but not making direct synapses with sensory afferents. Notably, it was shown that glutamate, like GABA, also causes PAD and consequent presynaptic inhibition of sensory transmission (63). The glutamate that mediates PAD was thought to be spillover from glutamatergic terminals located near the afferents. Thus, the rabies-labeled vGlut ${ }^{+}$spinal neurons in our study could represent such extrasynaptic glutamatergic inputs (Figure 7B). The second possibility is that some of the labeled neurons are postsynaptic to sensory afferents as a result of anterograde rabies spreading. This is similar to a previous report showing that rabies progressed from proprioceptive afferent neurons into postsynaptic motor neurons (25). We cannot rule out such anterograde spreading of rabies virus. However, several lines of evidence are consistent with a low probability of rabies anterograde spreading from cutaneous sensory afferents to spinal neurons. For example, although rabies virus infected approximately 1,300 Trpv1-Cre-expressing nociceptive neurons, they only spread into very few spinal neurons. In all tracing experiments $(n>40$
Trpv1-Cre and Avil-Cre mice examined), we never observed labeling of postsynaptic spinothalamic or spinoparabrachial projection neurons, whose axons cross the midline and enter the contralateral ventral funiculus. We never observed viral-labeled axon terminals in thalamus. Thus, at the most, only a small number of the labeled spinal neurons were postsynaptic targets of sensory afferents. Taken together, our results are consistent with the model that sensory afferents receive primarily GABAergic inputs (likely through axo-axonic synapses) and some extrasynaptic glutamatergic inputs from local interneurons in the spinal cord (Figure 7B). Some of the GABAergic interneurons coexpress glycine, but since DRG neurons do not express glycine receptor (64), glycine released from these neurons is unlikely to modulate primary afferents.

$R V M$-derived dual GABAergic/enkephalinergic inputs onto primary afferents have antinociceptive functions. Rabies labeling in RVM indicated this region as a major source of direct supra-spinal inputs to primary afferents. About $17 \%$ of labeled RVM-pre neurons were found to be 5-HT. RVM-derived 5-HT inputs are known to play an important facilitatory role in the development of pain hypersensitivity in chronic conditions (65). Our tracing results suggest that some of the descending 5-HT terminals are positioned near the primary afferents and could therefore directly modulate sensory transmission (Figure 7C). In agreement with this, a recent elegant study demonstrated that 5-HT directly sensitize central nociceptive terminals (66).

Interestingly, labeled RVM-pre neurons were predominantly (80\%) GABAergic/glycinergic, and the majority of GABAergic RVM-pre neurons also expressed the proenkephalin gene Penk1 (Figure 7C). Enkephalins are endogenous opioid ligands. Almost 40 years ago, Duggan et al. had already showed that application of enkephalins reduced the response of spinal neurons to noxious heat stimuli (67). Like GABA receptors, the MOR and $\delta$-opioid receptor (DOR) for enkephalins are found both on primary sensory afferents and on many spinal neurons (68-71). Thus, RVM-derived GABA and enkephalins probably function at both presynaptic and postsynaptic sites. Here, using an optogenetic approach to selectively activate the RVM-pre axons in spinal cord, we found that photostimulation induced DRPs with short latency, indicating that the RVM-pre neurons indeed can provide presynaptic inputs for primary afferents.

We assessed the function of the GABAergic/enkephalinergic RVM-pre neurons first using TeLC-mediated inhibition of synaptic vesicle release from RVM-pre neurons. Results showed behavioral hypersensitivity to heat, while the mechanical sensitivity to von Frey fiber was unchanged. These findings suggest that GABA released from RVM-pre is required to suppress hypersensitivity to noxious heat under normal conditions. Previously, tonic supraspinal descending modulation of PAD had been shown for group Ia afferent (72). Although there had not been such studies for cutaneous afferents, it is possible that GABA derived from RVM-pre neurons also tonically modulate cutaneous afferent transmission. What might explain the finding that, in the absence of RVMderived GABA, mechanical sensitivity is not affected? Basbaum, Scherrer, and their colleagues previously showed that DOR, which is the high-affinity receptor for enkephalin, is mainly expressed in nonpeptidergic and in myelinated sensory fibers, and DOR agonists reduce acute mechanical but not heat pain (73). They further 
showed that DOR presynaptically regulate mechanical inputs from sensory neurons to spinal cord (74). Thus, it is conceivable that DORs expressed on mechanosensitive fibers mediate the pain-suppressive effects of enkephalins released from the RVMpre neurons, even when RVM release of GABA is blocked.

When we eliminated RVM release of both GABA and enkephalin by ablating these neurons, mice behaviors showed hypersensitivity to both heat and von Frey mechanical stimulation. When we activated these RVM-pre neurons using a chemogenetic approach, both the heat and mechanical sensitivities are decreased (i.e., hyposensitive) in behavioral tests. Thus, the dual GABAergic-enkephalinergic RVM-pre neurons indeed function to suppress pain in adult mice. Previous studies showed that RVM contains 3 classes of neurons: ON-cells, OFF-cells, and NEUTRAL-cells, which are intermingled $(18,19,75)$. Since OFFcells have antinociceptive functions, some of the GABAergic/ enkephalinergic RVM-pre neurons might correspond to the canonical RVM OFF-cells. However, it was shown that RVM functions to facilitate pain in neonatal and young rats, and only switches to pain inhibition during peri-adolescence development; such switches depended on central opioid signaling (62). Thus, in early development, one cannot simply divide RVM neurons into ON- or OFFcells, as all cells appear to be pronociception (62). Future in vivo recording of optogenetically labeled RVM-pre neurons are needed to determine whether these neurons show OFF-responses in nociceptive behaviors in adult mice. Additionally, the RVM region was also found to regulate the autonomic system functions (76), and such neurons may not belong to the ON-, OFF-, or NEUTRALcell categories. Regardless of whether or not the dual GABAergic/ enkephalinergic RVM-pre neurons are OFF-cells, we now have a molecular identification of and a molecular tool (Lenti-Penk1Cre) to selectively study this specific class of antinociception RVM neurons. The new knowledge and the new tool pave the ways for future dissection of the circuits connected with these RVM-pre neurons and their roles under pathological pain conditions.

\section{Methods}

Mice. RФGT, Avil-Cre RФGT, Trpv1-Cre RФGT, and Trpv1-Cre RФGT RФtomato mice were used in this study; all alleles are heterozygous. Avil-Cre (29, 30), RФGT (27, 28), Trpv1-Cre (31), and ROSAФtomato mice (77) have all been described previously. WT C57BL/6 mice were purchased from Charles River Laboratories. Gad2-Cre mice were obtained from the Jackson Laboratory (stock number 010802).

Viruses. $\triangle \mathrm{G}-\mathrm{RV}-\mathrm{GFP}$ virus $\left(\sim 1 \times 10^{9} \mathrm{IFU} / \mathrm{ml}\right)$ was obtained from Salk Institute vector core. AAV5-EF1 $\alpha$-FLEX-taCasp3-TEVp virus was a gift from Nirao Shah, UCSF, San Francisco, California, USA. The construct pAAV-hSyn-FLEX-TeLC-P2A-GFP was a gift from Guoping Feng, MIT, Cambridge, Massachusetts, USA. AAV2-CAG-FLEX-GFP and AAVhSyn-DIO-hM3D $(\mathrm{Gq})$-mCherry were purchased from University of North Carolina at Chapel Hill vector core. AAV-EF1a-FLEX-hChR2eYFP was purchased from the University of Pennsylvania vector core.

EnvA-RV-GFP rabies viruses were produced and purified following the protocol from the Callaway Labs (78). First, SAD- $\triangle \mathrm{G}-\mathrm{GFP}$ rabies viruses (79) were expanded in B7GG cells (a gift from Edward Callaway, Salk Institute, La Jolla, California, USA), and subsequently, the virus containing culture supernatant was concentrated by centrifugation at 25,000 rpm with Beckman SW-28 rotor for 2 hours. The pellet was resuspended and used to infect the BHK-EnvA stable cell line (a gift from Edward Callaway, Salk Institute, La Jolla, California, USA). After a series of culture media collection, the virus-containing media were concentrated as above, resuspended in an appropriate volume, aliquoted, and stored at $-80^{\circ} \mathrm{C}$. The titer of EnvA-RV-GFP rabies viruses was estimated to be approximately $5 \times 10^{8} \mathrm{IFU} / \mathrm{ml}$.

For Lenti-Penk1-Cre virus, a conserved 726-bp genomic sequence just upstream of the first exon of the Penk1 gene was cloned and used as a promoter to drive Cre expression. Lentiviruses were packaged and produced in HEK293T cells (catalog CRL-11286, ATCC). In brief, HEK293T cells were transiently transfected with a mixture of pLentiPenk1-Cre, delta 8.9, and VSVG using polyethyleneimine "MAX" (PEI MAX) (catalog 24765, Polysciences Inc.). Forty-eight hours after transfection, the cell-culture media was collected and centrifuged at 3,000 rpm for 15 minutes, and the supernatant was filtered with $0.45 \mu \mathrm{m}$ filter unit (catalog SCHVU01RE, Millipore). The filtered virus-containing supernatant was loaded into the centrifuge tubes with $3 \mathrm{ml}$ of $20 \%$ sucrose cushion at the bottom and spun at 25,000 rpm with Beckman SW-28 rotor for 2 hours at $4^{\circ} \mathrm{C}$. The pellet was resuspended in an appropriate volume of PBS without calcium and magnesium $\left(-\mathrm{Ca}^{2+},-\mathrm{Mg}^{2+}\right)$, aliquoted, and stored at $-80^{\circ} \mathrm{C}$. The titer of Lenti-Penk1-Cre virus was estimated to be approximately $1 \times 10^{8} \mathrm{IFU} / \mathrm{ml}$.

For producing AAV8-hSyn-FLEX-GFP and AAV8-hSyn-FLEXTeLC-2A-GFP, HEK293T cells were transiently transfected with pAAVhSyn-FLEX-GFP or pAAV-hSyn-FLEX-TeLC-P2A-GFP, AAV serotype plasmid AAV8, and pAd.DELTA F6 using PEI MAX (catalog 24765, Polysciences Inc.). Seventy-two hours after transfection, the cell-culture media was discarded and the cells were collected in PBS, frozen, thawed 3 times using a dry ice/ethanol bath, and centrifuged at 3,000 rpm for 30 minutes. The contaminating DNA in the supernatant was removed by adding Benzonase and was incubated at $37^{\circ} \mathrm{C}$ for $30 \mathrm{~min}$ utes. The viral crude solution was filtered through $0.45 \mu \mathrm{m}$ filter (Millipore), and spun at 25,000 rpm for 90 minutes using a Beckman SW-28 rotor. The pellet was resuspended in $4 \mathrm{ml} \mathrm{PBS}$ and filtered through a $0.45-\mu \mathrm{m}$ filter and a $0.22-\mu \mathrm{m}$ filter sequentially. The viral crude solution was concentrated to 200-250 $\mu$ l using the Amicon Ultra-4 filter unit $(100 \mathrm{kDa})$, washed with $4 \mathrm{ml}$ PBS once, and concentrated to an appropriate volume. The viral solution was further purified using a $0.22-\mu \mathrm{m}$ Ultrafree-MC centrifugal filter unit (catalog UFC30GVOS, Millipore), and aliquots were stored at $-80^{\circ} \mathrm{C}$ until use.

Viral labeling experiments. All P1 pups were anesthetized by isoflurane and hypothermia and injected with about $200 \mathrm{nl}$ of $\Delta \mathrm{G}-\mathrm{RV}-\mathrm{GFP}$ $\left(\sim 1 \times 10^{9} \mathrm{IFU} / \mathrm{ml}\right)$ in the plantar skin of the right forepaw. Brains and spinal cords were collected 7 days after injection at P8. For silencing RVM inputs, either $1 \mu \mathrm{l}$ total of Lenti-Penk1-Cre/AAV-FLEX-TeLC-GFP (1:1 volume) or $1 \mu \mathrm{l}$ total of Lenti-Penk1-Cre/AAV-FLEX-taCasp3-TEVp (1:1 volume) were coinjected into RVM region of about 6- to 8-week-old WT C57BL/6 mice (coordinates from Bregma as following: anterio-posterior axis, $-5.5 \mathrm{~mm}$; lateral, $0.0 \mathrm{~mm}$; depths, $-5.85 \mathrm{~mm}$ ); coinjection of $1 \mu \mathrm{l}$ Lenti-Penk1-Cre and AAV-FLEX-GFP (1:1 volume) into RVM regions were used as the control. Similarly, $500 \mathrm{nl}$ AAV-hSyn-FLEX-TeLC-GFP alone was injected into RVM region of Gad2-Cre mice. For recording DRPs, $1 \mu \mathrm{l}$ total of Lenti-Penk1-Cre/AAV-EF1a-FLEX-hChR2-eYFP (1:1 volume) were coinjected into RVM region of about 6- to 8-week-old WT C57BL/ 6 mice. For activating RVM inputs, $1 \mu \mathrm{l}$ total of Lenti-Penk1-Cre/ AAV-hSyn-DIO-hM3D(Gq)-mCherry (1:1 volume) were coinjected into RVM region of about 6- to 8-week-old WT C57BL/6 mice. 
In situ hybridization and immunostaining. Immunostaining and in situ hybridization were performed according to standard protocols (28). Briefly, Digoxigenin-labeled (DIG-labeled) or FITC-labeled RNA probes were used for in situ hybridizations. For antisense probe, the mouse cDNA fragment was amplified by PCR with the antisense primer containing the $\mathrm{T} 7$ promoter sequence. In vitro transcription was then performed from the PCR-amplified template using T7 RNA polymerase (catalog 10881767001, Roche Applied Science) with DIG-UTP (catalog 11277073910, Roche Applied Science) or fluorescein-UTP (catalog 11685619910, Roche Applied Science) for the synthesis of the antisense probes. Sections $(20 \mu \mathrm{m})$ were used for in situ hybridization. Prehybridization, hybridization, and washing were performed according to standard methods. Sections were then incubated with alkaline phosphatase-conjugated anti-DIG (1:3,500; catalog 11093274910, Roche Applied Science) or POD anti-FITC (1:500; catalog 11426346910, Roche Applied Science). After washing, the in situ signals were developed with Fast Red substrate (catalog 11496549001, Roche Applied Science) for DIG-labeled RNA probes or FITC-TSA (catalog NEL741001KT, PerkinElmer) for FITClabeled RNA probes. Following in situ hybridizations, GFP signals were developed with rabbit anti-GFP (1:1,000; catalog ab290, Abcam) for overnight at $4^{\circ} \mathrm{C}$ and then detected with Alexa Fluor 488 anti-mouse IgG (1:1,000; catalog A11008, Molecular Probes). In addition, guinea pig anti-vGlut1 (1:1,000; catalog AB5905, Millipore), rabbit CGRP (1:1,000; catalog T-4239, Peninsula Laboratories International Inc.), and GS-IB4-Alexa 488-conjugated (1:1,000; catalog 121411, Invitrogen) were also used.

Extracellular recording of photostimulation evoked DRPs. Four weeks after coinjection of Lenti-Penk1-Cre and AAV-EF1a-FLEXhChR2-eYFP into RVM of adult male mice, the animals were anesthetized with isofluorane, transcardially perfused in ice-cold NMDG artificial cerebrospinal fluid (NMDG-ACSF; containing $92 \mathrm{mM} \mathrm{NMDG}$, $2.5 \mathrm{mM} \mathrm{KCl}, 1.2 \mathrm{mM} \mathrm{NaH}_{2} \mathrm{PO}_{4}, 30 \mathrm{mM} \mathrm{NaHCO}{ }_{3}, 20 \mathrm{mM}$ HEPES, 2 $\mathrm{mM}$ glucose, $5 \mathrm{mM}$ sodium ascorbate, $2 \mathrm{mM}$ thiourea, $3 \mathrm{mM}$ sodium pyruvate, $10 \mathrm{mM} \mathrm{MgSO}_{4}$, and $0.5 \mathrm{mM} \mathrm{CaCl}_{2}$ ), and bubbled with $5 \%$ $\mathrm{CO}_{2} / 95 \% \mathrm{O}_{2}$. The cervical vertebral column was then extracted and placed in a dissection dish containing ice-cold oxygenated NMDGACSF and resting in a small container of ice. Using No. 5 forceps and fine scissors (FST), the dura was longitudinally hemisected and peeled off of the ventral surface on the right and left sides of the spinal cord. Care was taken not to damage the dorsal roots. The spinal cord was hemisected by a beveled crescent scalpel through the midline. Tissues were then bubbled in same solution at $37^{\circ} \mathrm{C}$ for 8 minutes and transferred to bubbled, modified-HEPES ACSF at $23^{\circ} \mathrm{C}-25^{\circ} \mathrm{C}(92 \mathrm{mM} \mathrm{NaCl}$, $2.5 \mathrm{mM} \mathrm{KCl}, 1.2 \mathrm{mM} \mathrm{NaH}_{2} \mathrm{PO}_{4}, 30 \mathrm{mM} \mathrm{NaHCO}{ }_{3}, 20$ mM HEPES, 2 $\mathrm{mM}$ glucose, $5 \mathrm{mM}$ sodium ascorbate, $2 \mathrm{mM}$ thiourea, $3 \mathrm{mM}$ sodium pyruvate, $2 \mathrm{mM} \mathrm{MgSO}_{4}$, and $2 \mathrm{mM} \mathrm{CaCl}_{2}$ ) for at least 1 hour before recording. Recordings were performed in submerged chamber, superfused with continuously bubbled ACSF $(125 \mathrm{mM} \mathrm{NaCl}, 2.5 \mathrm{mM} \mathrm{KCl}$,
$1.25 \mathrm{mM} \mathrm{NaH}_{2} \mathrm{PO}_{4}, 26 \mathrm{mM} \mathrm{NaHCO}_{3}, 20 \mathrm{mM}$ glucose, $2 \mathrm{mM} \mathrm{CaCl}_{2}$, and $1.3 \mathrm{mM} \mathrm{MgSO}_{4}$ ) at $2-5 \mathrm{ml} \mathrm{min}^{-1}$ at $23^{\circ} \mathrm{C}-25^{\circ} \mathrm{C}$. The hemisected spinal cord was placed in the chamber; the ventral surface was secured with a harp with fine, loose, nylon fibers. Dorsal roots were placed into suction electrodes. Extracellular recordings were obtained from the dorsal roots in response to light activation of ChR2-expressing axons from RVM. Signals were amplified with Multiclamp 700B (filtered at $2 \mathrm{kHz}$ ), digitized with Digidata 1440A (5 kHz), and recorded using pClamp 10 software (Axon). Photostimulation was performed using a $473 \mathrm{~nm}$ LED (CoolLED, pE4000) controlled by pClamp 10 software (Axon). Light pulse width was $50 \mathrm{~ms}$.

All electrophysiology data were analyzed and filtered off-line using Neuromatic package (Think Random) in Igor Pro software (WaveMetrics). Off-line analysis was performed by averaging 3 traces. Light-evoked EPSP peak amplitude and latency were analyzed. The latency of the light-evoked DRPs was defined as the time from the onset of the stimulus to the first measurable deflection of the potential from the baseline.

Behavioral tests. Male mice about 6-8 weeks old were used for all viral injections and subsequent behavior tests. Thermal sensitivity was tested using a hot plate at $50^{\circ} \mathrm{C}$ and tail-flick test, expressed as paw-withdrawal latency and tail-flick latency. For testing mechanical sensitivity after viral injection into RVM regions, the plantar surface of the hindpaw was stimulated with a series of von Frey hairs $(0.008-2 \mathrm{~g}$, Stoelting), and the $50 \%$ paw-withdrawal threshold was determined using Dixon's up-down method. For DREADD experiments, CNO (Sigma-Aldrich) was administrated at $0.3 \mathrm{mg} / \mathrm{kg}$ by i.p. injection. Behavioral tests were conducted between 30 minutes to 2 hours following administration of either saline or CNO. All behaviors were performed with genotypes blind to the experimenter.

Statistics. All data were expressed as mean \pm SEM. Behavioral data were analyzed using 2-tailed Student's $t$ test between 2 groups (experimental or control), or in the case of multiple groups, one-way ANOVA followed by post-hoc Bonferroni test. The criterion for statistical significance was $P<0.05$.

Study approval. All experimental protocols were approved by the Duke University Institutional Animal Care and Use Committee.

\section{Acknowledgments}

We thank Mark Hoon at NIDCR for the Trpv1-Cre mouse line. We thank Ru-rong Ji, Richard Mooney, and Steve Lisberger, as well as members of the Wang lab, for critical reading of the manuscript. This work is supported by NIH grants DE019440 and NS077986 to F. Wang.

Address correspondence to: Fan Wang, Department of Neurobiology, Duke University Medical Center, Box 3209, Durham, North Carolina 27710, USA. USA. Phone: 919.684.3682; E-mail: fan. wang@duke.edu.
1. Prescott SA, Ratte S. Pain processing by spinal microcircuits: afferent combinatorics. Curr Opin Neurobiol. 2012;22(4):631-639.

2. Basbaum AI, Bautista DM, Scherrer G, Julius D. Cellular and molecular mechanisms of pain. Cell. 2009;139(2):267-284.

3. Braz J, Solorzano C, Wang X, Basbaum AI. Trans- mitting pain and itch messages: a contemporary view of the spinal cord circuits that generate gate control. Neuron. 2014;82(3):522-536.

4. Todd AJ. Neuronal circuitry for pain processing in the dorsal horn. Nat Rev Neurosci. 2010;11(12):823-836.

5. Ribeiro-da-Silva A, Coimbra A. Two types of synaptic glomeruli and their distribution in laminae I-III of the rat spinal cord. J Comp Neurol. 1982;209(2):176-186.

6. Rethelyi M, Light AR, Perl ER. Synaptic complexes formed by functionally defined primary afferent units with fine myelinated fibers. J Comp Neurol.1982;207(4):381-393. 
7. Todd AJ. GABA and glycine in synaptic glomeruli of the rat spinal dorsal horn. Eur J Neurosci. 1996;8(12):2492-2498.

8. Rudomin P, Engberg I, Jimenez I. Mechanisms involved in presynaptic depolarization of group I and rubrospinal fibers in cat spinal cord. J Neurophysiol. 1981;46(3):532-548.

9. Kullmann DM, Ruiz A, Rusakov DM, Scott R, Semyanov A, Walker MC. Presynaptic, extrasynaptic and axonal GABAA receptors in the CNS: where and why? Prog Biophys Mol Biol. 2005;87(1):33-46.

10 . Witschi $\mathrm{R}$, et al. Presynaptic $\alpha 2$-GABAA receptors in primary afferent depolarization and spinal pain control. JNeurosci. 2011;31(22):8134-8142.

11. Wall PD. The laminar organization of dorsal horn and effects of descending impulses. J Physiol. 1967;188(3):403-423.

12. Oliveras JL, Besson JM, Guilbaud G, Liebeskind JC. Behavioral and electrophysiological evidence of pain inhibition from midbrain stimulation in the cat. Exp Brain Res. 1974;20(1):32-44.

13. Oliveras JL, Redjemi F, Guilbaud G, Besson JM. Analgesia induced by electrical stimulation of the inferior centralis nucleus of the raphe in the cat. Pain. 1975;1(2):139-145.

14. Reynolds DV. Surgery in the rat during electrical analgesia induced by focal brain stimulation. Science. 1969;164(3878):444-445.

15. Liebeskind JC, Guilbaud G, Besson JM, Oliveras JL. Analgesia from electrical stimulation of the periaqueductal gray matter in the cat: behavioral observations and inhibitory effects on spinal cord interneurons. Brain Res. 1973;50(2):441-446.

16. Heinricher MM, Tavares I, Leith JL, Lumb BM. Descending control of nociception: Specificity, recruitment and plasticity. Brain Res Rev. 2009;60(1):214-225.

17. Ossipov MH, Dussor GO, Porreca F. Central modulation of pain. J Clin Invest. 2010;120(11):3779-3787.

18. Fields HL, Bry J, Hentall I, Zorman G. The activity of neurons in the rostral medulla of the rat during withdrawal from noxious heat. J Neurosci. 1983;3(12):2545-2552.

19. Mason P. Central mechanisms of pain modulation. Curr Opin Neurobiol. 1999;9(4):436-441.

20. Gebhart GF. Descending modulation of pain. Neurosci Biobehav Rev. 2004;27(8):729-737.

21. Budai D, Fields HL. Endogenous opioid peptides acting at mu-opioid receptors in the dorsal horn contribute to midbrain modulation of spinal nociceptive neurons. J Neurophysiol. 1998;79(2):677-687.

22. Wickersham IR, et al. Monosynaptic restriction of transsynaptic tracing from single, genetically targeted neurons. Neuron. 2007;53(5):639-647.

23. Callaway EM. Transneuronal circuit tracing with neurotropic viruses. Curr Opin Neurobiol. 2008;18(6):617-623.

24. Tsiang H, Lycke E, Ceccaldi PE, Ermine A, Hirardot $\mathrm{X}$. The anterograde transport of rabies virus in rat sensory dorsal root ganglia neurons. J Gen Virol. 1989;70(pt 8):2075-2085.

25. Zampieri N, Jessell TM, Murray AJ. Mapping sensory circuits by anterograde transsynaptic transfer of recombinant rabies virus. Neuron. 2014;81(4):766-778.
26. Coulon P, Derbin C, Kucera P, Lafay F, Prehaud $\mathrm{C}$, Flamand A. Invasion of the peripheral nervous systems of adult mice by the CVS strain of rabies virus and its avirulent derivative AvO1. JVirol. 1989;63(8):3550-3554.

27. Stanek E IV, Cheng S, Takatoh J, Han B-X, Wang F. Monosynaptic premotor circuit tracing reveals neural substrates for oro-motor coordination. Elife. 2014;3:e02511.

28. Takatoh J, et al. New modules are added to vibrissal premotor circuitry with the emergence of exploratory whisking. Neuron. 2013;77(2):346-360.

29. da Silva S, et al. Proper formation of whisker barrelettes requires periphery-derived Smad4-dependent TGF- $\beta$ signaling. Proc Natl Acad Sci U S A. 2011;108(8):3395-3400.

30. Zhou X, et al. Deletion of PIK3C3/Vps34 in sensory neurons causes rapid neurodegeneration by disrupting the endosomal but not the autophagic pathway. Proc Natl Acad Sci U S A. 2010;107(20):9424-9429.

31. Mishra SK, Tisel SM, Orestes P, Bhangoo SK, Hoon MA. TRPV1-lineage neurons are required for thermal sensation. $E M B O J$. 2011;30(3):582-593.

32. Ribeiro-da-Silva A, Tagari P, Cuello AC. Morphological characterization of substance P-like immunoreactive glomeruli in the superficial dorsal horn of the rat spinal cord and trigeminal subnucleus caudalis: a quantitative study. J Comp Neurol. 1989;281(4):497-515.

33. Ugolini G. Advances in viral transneuronal tracing. J Neurosci Methods. 2010;194(1):2-20.

34. Rudy B, Fishell G, Lee S, Hjerling-Leffler J. Three groups of interneurons account for nearly 100\% of neocortical GABAergic neurons. Dev Neurobiol. 2011;71(1):45-61.

35. Duan B, et al. Identification of spinal circuits transmitting and gating mechanical pain. Cell. 2014;159(6):1417-1432.

36. Huang J, et al. Neurochemical properties of enkephalinergic neurons in lumbar spinal dorsal horn revealed by preproenkephalin-green fluorescent protein transgenic mice. J Neurochem. 2010;113(6):1555-1564

37. Sardella TC, Polgar E, Watanabe M, Todd AJ. A quantitative study of neuronal nitric oxide synthase expression in laminae I-III of the rat spinal dorsal horn. Neuroscience. 2011;192:708-720.

38. Watkins LR, Suberg SN, Thurston CL, Culhane ES. Role of spinal cord neuropeptides in pain sensitivity and analgesia: thyrotropin releasing hormone and vasopressin. Brain Res. 1986;362(2):308-317.

39. Freire MA, Guimaraes JS, Leal WG, Pereira A. Pain modulation by nitric oxide in the spinal cord. Front Neurosci. 2009;3(2):175-181.

40. Fields H. State-dependent opioid control of pain Nat Rev Neurosci. 2004;5(7):565-575.

41. Marinelli S, Vaughan CW, Schnell SA, Wessendorf MW, Christie MJ. Rostral ventromedial medulla neurons that project to the spinal cord express multiple opioid receptor phenotypes. J Neurosci. 2002;22(24):10847-10855.

42. Antal M, Petko M, Polgar E, Heizmann CW, Storm-Mathisen J. Direct evidence of an extensive GABAergic innervation of the spi- nal dorsal horn by fibres descending from the rostral ventromedial medulla. Neuroscience. 1996;73(2):509-518.

43. Fields HL, Malick A, Burstein R. Dorsal horn projection targets of $\mathrm{ON}$ and OFF cells in the rostral ventromedial medulla. J Neurophysiol. 1995;74(4):1742-1759.

44. Mason P, Fields HL. Axonal trajectories and terminations of on- and off-cells in the cat lower brainstem. J Comp Neurol. 1989;288(2):185-207.

45. Adamantidis AR, Zhang F, de Lecea L, Deisseroth K. Optogenetics: opsins and optical interfaces in neuroscience. Cold Spring Harb Protoc. 2014;2014(8):815-822.

46. Rudomin P, Schmidt RF. Presynaptic inhibition in the vertebrate spinal cord revisited. Exp Brain Res. 1999;129(1):1-37.

47. Alvarez-Leefmans FJ. Chloride transporters in presynaptic inhibition, pain and neurogenic inflammation. In: Alvarez-Leefmans F, Delpire E, eds. Physiology and Pathology of Chloride Transporters and Channels in the Nervous System: From Molecules to Diseases. San Diego, California, USA: Elsevier-Academic Press; 2009:439-470.

48. Link E, et al. Tetanus toxin action: inhibition of neurotransmitter release linked to synaptobrevin proteolysis. Biochem Biophys Res Commun. 1992;189(2):1017-1023.

49. Schiavo G, et al. Tetanus and botulinum-B neurotoxins block neurotransmitter release by proteolytic cleavage of synaptobrevin. Nature. 1992;359(6398):832-835.

50. Navone F, Di Gioia G, Jahn R, Browning M, Greengard P, De Camilli P. Microvesicles of the neurohypophysis are biochemically related to small synaptic vesicles of presynaptic nerve terminals. J Cell Biol. 1989;109(6 pt 2):3425-3433.

51. Sweeney ST, Broadie K, Keane J, Niemann H, O'Kane CJ. Targeted expression of tetanus toxin light chain in Drosophila specifically eliminates synaptic transmission and causes behavioral defects. Neuron. 1995;14(2):341-351.

52. Jones BE, Holmes CJ, Rodriguezveiga E, Mainville L. GABA-synthesizing neurons in the medulla: their relationship to serotonin-containing and spinally projecting neurons in the rat. J Comp Neurol. 1991;313(2):349-367.

53. Yang CF, et al. Sexually dimorphic neurons in the ventromedial hypothalamus govern mating in both sexes and aggression in males. Cell. 2013;153(4):896-909.

54. Alexander GM, et al. Remote control of neuronal activity in transgenic mice expressing evolved G protein-coupled receptors. Neuron. 2009;63(1):27-39.

55. Schnell MJ, McGettigan JP, Wirblich C, Papaneri A. The cell biology of rabies virus: using stealth to reach the brain. Nat Rev Microbiol. 2010;8(1):51-61.

56. Pagadala P, et al. Loss of NR1 subunit of NMDARs in primary sensory neurons leads to hyperexcitability and pain hypersensitivity: involvement of $\mathrm{Ca}(2+)$-activated small conductance potassium channels. J Neurosci. 2013;33(33):13425-13430.

57. Kung LH, et al. Evidence for glutamate as a neuroglial transmitter within sensory ganglia. PLoS One. 2013;8(7):e68312. 
58. Bráz JM, Ackerman L, Basbaum AI. Sciatic nerve transection triggers release and intercellular transfer of a genetically expressed macromolecular tracer in dorsal root ganglia. J Comp Neurol. 2011;519(13):2648-2657.

59. Zhang X, Chen Y, Wang C, Huang LY. Neuronal somatic ATP release triggers neuron-satellite glial cell communication in dorsal root ganglia. Proc Natl Acad Sci U S A . 2007;104(23):9864-9869.

60. Ceruti S, et al. Calcitonin gene-related peptidemediated enhancement of purinergic neuron/ glia communication by the algogenic factor bradykinin in mouse trigeminal ganglia from wild-type and R192Q Cav2.1 Knock-in mice: implications for basic mechanisms of migraine pain. J Neurosci. 2011;31(10):3638-3649.

61. Purkiss J, Welch M, Doward S, Foster K. Capsaicin-stimulated release of substance $\mathrm{P}$ from cultured dorsal root ganglion neurons: involvement of two distinct mechanisms. Biochem Pharmacol. 2000;59(11):1403-1406.

62. Hathway GJ, Vega-Avelaira D, Fitzgerald M. A critical period in the supraspinal control of pain: Opioid-dependent changes in brainstem rostroventral medulla function in preadolescence. Pain. 2012;153(4):775-783.

63. Lee CJ, et al. Functional expression of AMPA receptors on central terminals of rat dorsal root ganglion neurons and presynaptic inhibition of glutamate release. Neuron. 2002;35(1):135-146.

64. Lorenzo LE, et al. Gephyrin clusters are absent from small diameter primary afferent terminals despite the presence of $\mathrm{GABA}(\mathrm{A})$ receptors. J Neurosci. 2014;34(24):8300-8317.

65. Wei F, et al. Molecular depletion of descending serotonin unmasks its novel facilitatory role in the development of persistent pain. JNeurosci. 2010;30(25):8624-8636.

66. Kim YS, et al. Central terminal sensitization of TRPV1 by descending serotonergic facilitation modulates chronic pain. Neuron. 2014;81(4):873-887.

67. Duggan AW, Hall JG, Headley PM. Morphine, enkephalin and the substantia gelatinosa. Nature. 1976;264(5585):456-458.

68. Besse D, Lombard MC, Besson JM. Autoradiographic distribution of $\mathrm{mu}$, delta and kappa opioid binding sites in the superficial dorsal horn, over the rostrocaudal axis of the rat spinal cord. Brain Res. 1991;548(1-2):287-291.

69. Arvidsson U, et al. delta-Opioid receptor immunoreactivity: distribution in brainstem and spinal cord, and relationship to biogenic amines and enkephalin. J Neurosci. 1995;15(2):1215-1235.

70. Arvidsson U, et al. Distribution and targeting of a mu-opioid receptor (MOR1) in brain and spinal cord. J Neurosci. 1995;15(5 pt 1):3328-3341.

71. Mansour A, Fox CA, Burke S, Akil H, Watson SJ. Immunohistochemical localization of the cloned mu opioid receptor in the rat CNS. JChem Neuroanat. 1995;8(4):283-305.

72. Rudomin P, Lomeli J, Quevedo J. Tonic differen- tial supraspinal modulation of PAD and PAH of segmental and ascending intraspinal collaterals of single group I muscle afferents in the cat spinal cord. Exp Brain Res. 2004;159(2):239-250.

73. Scherrer G, et al. Dissociation of the opioid receptor mechanisms that control mechanical and heat pain. Cell. 2009;137(6):1148-1159.

74. Bardoni R, et al. Delta opioid receptors presynaptically regulate cutaneous mechanosensory neuron input to the spinal cord dorsal horn. Neuron. 2014;81(6):1312-1327.

75. Fields HL, Bry J, Hentall I, Zorman G. The activity of neurons in the rostral medulla of the rat during withdrawal from noxious heat. J Neurosci. 1983;3(12):2545-2552.

76. Cerri M, et al. The inhibition of neurons in the central nervous pathways for thermoregulatory cold defense induces a suspended animation state in the rat. J Neurosci. 2013;33(7):2984-2993.

77. Arenkiel BR, et al. Activity-induced remodeling of olfactory bulb microcircuits revealed by monosynaptic tracing. PLoS One. 2011;6(12):e29423.

78. Osakada F, Callaway EM. Design and generation of recombinant rabies virus vectors. Nat Protoc. 2013;8(8):1583-1601.

79. Wall NR, Wickersham IR, Cetin A, De La Parra M, Callaway EM. Monosynaptic circuit tracing in vivo through Cre-dependent targeting and complementation of modified rabies virus. Proc Natl Acad Sci U S A. 2010;107(50):21848-21853. 